\title{
SIMULATING COMPLEX SOCIAL-BEHAVIORAL SYSTEMS
}

\author{
Kathleen M. Carley \\ Institute for Software Research \\ Carnegie Mellon University \\ 5000 Forbes Ave, Pittsburgh PA, USA \\ and \\ Netanomics \\ 1924 Glen Mitchell Rd, Sewickley PA, USA \\ kathleen.carley@cs.cmu.edu
}

\begin{abstract}
Social-behavioral systems are complex. Consequently, they are often assessed using simulation. Particularly valuable is the artificial society approach, in which the social-behavioral system is examined using an agent-based dynamic-network simulation. Historically such models were far removed from real-world systems, costly to build, difficult to maintain, and costly to validate. While these problems still exist, there are practices that, if followed, reduce these problems, support model re-uses, and increase model scalability and veridicality. The practices are predicated on the assumption that context matters. Consequently the purpose of the simulation drives how the model is built, analyzed and validated. These practices are rooted in the unalterable fact that human systems are distinct from physical and mechanical systems and so require fundamentally different types of, and guidelines for, modeling and validation.
\end{abstract}

Keywords: dynamic networks, agent-based modeling, social cognition, validation.

\section{INTRODUCTION}

Social-behavioral systems are classic examples of complex systems. Complex systems are often characterized as having a large number of parts that interact in non-linear ways. In such systems the aggregate or group behavior is not derivable from the summation or averaging of the activity of the individual components; e.g., a group's decision is generally more extreme than the decisions of the individuals in the group if taken separately. Further, in such systems there are typically multiple metastable states where a small change in starting conditions can result in major change in the overall system.

Social-behavioral systems are a special case of complex systems. They exhibit all the behaviors just discussed and more. This is because in a social complex system the key components are people, decision makers, or human groups. Social systems represent a special case of complexity because humans can learn. Models of physical systems, even complex systems models, such as those of sand piles or crystalline growth, are composed of components who interact in non-linear ways but those processes are stationary. In contrast, when humans are involved, the underlying processes themselves change. Second, learning processes and human growth literally mean that people will not always react to the exact same stimuli in the same way as the people themselves will be different. A third difference with complex physical systems is that human systems are composed of heterogeneous agents; whereas, physical systems can be composed of homogeneous agents. A fourth difference is that human systems generate collective-collaborativecompetitive artifacts that survive and evolve even when all original members are gone. Organizations and 
governments, e.g., can have a lifetime beyond that of their founders. Moreover those artifacts constrain and enable individual learning allowing for second order learning. Hence, social behavioral systems are not only complex, but it is a more adaptive creative complexity than that in physical complex systems. This can be thought of as super-complexity. A fifth difference is that in social behavioral systems there are typically multiple meta-stable processes where a small change in what processes are present or the way the processes are connected can result in major changes in individual behavior and social outcomes.

A key method for reasoning about complexity is simulation, particularly for reasoning about complex sociotechnical or social-behavioral systems (Edmonds, 2005). The use of these models and their viability has increased due to recent advances in statistics, the increased amount of digitized data, the spread of simulation toolkits, and the ease of getting access to high-speed multi-processor computers with large data storage. For simulation, agent-based (e.g., Bonabeau, 2002; vanDam et al, 2012; Davidsson, 2002), agentbased dynamic-network models (e.g., Carley and Morgan, 2016; Morgan et al, 2017), event-history (e.g., Box-Steffensmeie \& Jones, 2004) and system dynamic models (e.g., Sterman, 2001; Mohaghegh \& Mosleh, 2009) are generally used; however, no one simulation methodology is proving adequate.

Herein the focus is on agent-based models (ABM) of social-behavioral systems; while recognizing, that ABMs are often part of a hybrid model where the ABM is integrated with an event-based or network-based model. ABMs are particularly interesting from a complexity perspective as the key elements that cause social simulations to exhibit super complexity are clearly present and critical to the way ABMs are used. These elements are non-stationarity of process, agent leaning, agent heterogeneity, adaptive constraining artifacts, and meta-stable processes. Despite their growth in popularity, and the increasing number of modeling platforms such as NetLogo, MASON and Repast, ABMs remain costly to build, validate and use. Most ABMs are never completely empirically validated, though some form of face validity is generally done. Analyses are often misleading, particularly when the analysis does not consider the effect of replication or error propagation among sub-model components. Model re-use is rare. And so on.

Most of the scientific understanding of how to design, analyze and validate simulation models has been predicated on models of physical systems. As noted above, social systems are different. These differences are the fundamental reasons why simulations of social behavior often seem less "scientific" than simulations of physical systems and why models of human activity cannot be validated using traditional methods. Thus, the question is, "How can we put the simulation of social systems, and so the use of artificial societies, on a firmer scientific basis so as to support improved policy decisions and scientific progress?" To address this issue, this paper describes a set of best practices that are critical to the development of viable and useful agent-based simulations of complex social systems. These practices were selected as they support the development of a scientific foundation, contribute to model reuse or scalability, and are focused around using simulation models to reason about possible futures. Additional guidelines and alternative points of view appear in Davis et al, 2019 and Diallo et al, 2019 where other forms of models are discussed.

This assessment is predicated on the following guiding design principles:

- Model design and development

$\circ$ Purpose based modeling. You can't model everything, so what you model should be relevant to the scientific or policy exploration.

- Tradeoff based modeling. In an agent based model, there is generally a tradeoff between agent complexity and the number of agents that can be simultaneously active in a simulation. In other words, there is a cognitive/social complexity tradeoff.

- Model use and result interpretation

$\circ$ Non-stationarity, individual learning and group artifacts. Social systems are fundamentally different than physical systems, because people learn and adapt, processes are nonstationary, people create adaptive artifacts resulting in second order learning and so on.

- Meta-Stable processes. Adding in new elements or modules into a model may make it impossible to regenerate earlier results.

- Agent heterogeneity. Fundamental agent variation is critical for realistic outcomes. 
- Space of possibilities. ABMs and artificial societies are not designed for classifying data into categories but for exploring the set of possibilities, the paths that lead to those diverse outcomes, and to engage in counter-factual reasoning to support planning.

These basic principles have implications for building, analyzing the results from and validating simulation models. These principles, however, must be interpreted in terms of an additional overarching factor context (Burton and Obel 1995).

Context refers to the model's purpose and its subject domain. The purpose will dictate how detailed, accurate and validated the model needs to be. Assessing a model and its development and use cycle without context information tends to result in judgement errors about the model's utility. The subject domain, and the questions the model is being used to address, will dictate the quantity and quality of data available for instantiating and validating the model, the extent to which the internal processes are understood, the model's scope and so the need for scalability, and the likelihood of re-use and so the need for generalizability. Context needs to be clearly defined and used to guide model development, analysis and validation.

\section{TOWARD REUSABLE CONTEXT SENSITIVE MODELS}

Artificial societies can be simulated using agent-based dynamic-network models. While such simulations can be complex to develop, analyze and validate - there are practices that can afford better outcomes. Given the foregoing assumptions, a set of best-practices were identified - see Table 1. Herein, such practices that have been shown to work for large-scale realistic models capable of supporting policy decisions are discussed. These practices were known to work if they aided the development and use of the BioWar system for modeling the spread of weaponized and non-weaponized diseases (Carley et al, 2006), the Construct model used for exploring the co-evolution of social and cultural systems and reused in many domains including tax payment (Carley \& Maxwell, 2006), social adaptation (Carley et al, 2009), and nuclear deterrence (Lanham et al, 2011), and a model of state level socio-technical influence used for assessing the desire of nation states for acquiring weapons of mass destruction (Mezzour et al, 2018). These best practices were also informed by the review of other agent-based models.

Table 1: Relation of Best Practices to Model Design and Development Assumptions.

\begin{tabular}{|l|l|l|}
\hline \multicolumn{1}{|c|}{ Purpose based modeling } & \multicolumn{1}{c|}{ Tradeoff based modeling } \\
networks & $\begin{array}{l}\text { Purpose defines the time frame and hence } \\
\text { whether networks should be dynamic }\end{array}$ & $\begin{array}{l}\text { Realism impacts the effect of tradeoff on } \\
\text { computational requirements, and number of agents }\end{array}$ \\
\hline $\begin{array}{l}\text { Social } \\
\text { cognition }\end{array}$ & $\begin{array}{l}\text { Purpose defines the granularity of groups } \\
\text { and hence which aspects of social } \\
\text { cognition should be modeled }\end{array}$ & $\begin{array}{l}\text { Realism impacts the effect of tradeoff on } \\
\text { computational requirements, and number of agents }\end{array}$ \\
\hline $\begin{array}{l}\text { Measurable } \\
\text { parameters }\end{array}$ & Purpose defines parameters of concern & $\begin{array}{l}\text { Parameters, particularly the ratio of parameters } \\
\text { may impact these tradeoffs }\end{array}$ \\
\hline $\begin{array}{l}\text { Virtual } \\
\text { experiments }\end{array}$ & $\begin{array}{l}\text { Purpose defines virtual experiment and } \\
\text { virtual experiment keeps modeler focused }\end{array}$ & $\begin{array}{l}\text { Choices on these tradeoffs effect what virtual } \\
\text { experiments are possible }\end{array}$ \\
\hline $\begin{array}{l}\text { Interoperability } \\
\text { Purpose defines whether a model needs } \\
\text { to be interoperable }\end{array}$ & $\begin{array}{l}\text { Choices on these tradeoffs may constrain or } \\
\text { increase the ease of interoperation }\end{array}$ \\
\hline $\begin{array}{l}\text { Response } \\
\text { surface } \\
\text { methodology }\end{array}$ & $\begin{array}{l}\text { Creates a fast variant that can be used } \\
\text { within a limited region }\end{array}$ & $\begin{array}{l}\text { Creates a fast variant that can be used for a } \\
\text { specific tradeoff choice }\end{array}$ \\
\hline $\begin{array}{l}\text { Validation in } \\
\text { parts }\end{array}$ & Validation level depends on purpose & $\begin{array}{l}\text { Validation level and type depends on tradeoff } \\
\text { choice }\end{array}$ \\
\hline
\end{tabular}

Several of these practices speak to the design and building of a model: dynamic networks, social cognition, and measurable parameters. Other practices speak to the process of model use and result interpretation: virtual experiments, interoperability, response surface methodology, and validation in parts. Each of these practices bears on the over-riding assumptions previously identified. This was illustrated in Table 1. In 
addition, each practice impacts model use and interpretation. See Table 2 . The items shown in these tables are meant to be illustrative of the relation between best practice and assumption and not exhaustive.

Table 2: Relation of Best Practices to Model Use and Result Interpretation.

\begin{tabular}{|c|c|c|c|c|}
\hline & $\begin{array}{l}\text { Non-stationarity, } \\
\text { individual learning } \\
\text { and group artifacts }\end{array}$ & $\begin{array}{l}\text { Meta-Stable } \\
\text { processes }\end{array}$ & Agent heterogeneity & Space of possibilities \\
\hline $\begin{array}{l}\text { Dynamic } \\
\text { networks }\end{array}$ & $\begin{array}{l}\text { Leaning, adaptation } \\
\text { and process impact } \\
\text { dynamics }\end{array}$ & $\begin{array}{l}\text { Process impacts } \\
\text { dynamics }\end{array}$ & $\begin{array}{l}\text { Agents vary in } \\
\text { network position, and } \\
\text { network membership }\end{array}$ & $\begin{array}{l}\text { Networks constrains } \\
\text { and enables what is } \\
\text { possible }\end{array}$ \\
\hline $\begin{array}{l}\text { Social } \\
\text { cognition }\end{array}$ & $\begin{array}{l}\text { Realism impacts the } \\
\text { learning and } \\
\text { adaptation process }\end{array}$ & $\begin{array}{l}\text { Process impacts } \\
\text { social cognition }\end{array}$ & $\begin{array}{l}\text { Agents vary in what } \\
\text { they remember due to } \\
\text { social cognition }\end{array}$ & $\begin{array}{l}\text { Social cognition } \\
\text { constrains and } \\
\text { enables behavior }\end{array}$ \\
\hline $\begin{array}{l}\text { Measurable } \\
\text { parameters }\end{array}$ & $\begin{array}{l}\text { Parameters can } \\
\text { constrain how } \\
\text { learning and } \\
\text { adaptation operate }\end{array}$ & $\begin{array}{l}\text { Parameters can } \\
\text { constrain which } \\
\text { processes are used } \\
\text { and in which order }\end{array}$ & $\begin{array}{l}\text { Parameters can } \\
\text { constrain or enhance } \\
\text { agent heterogeneity }\end{array}$ & $\begin{array}{l}\text { Parameters are key to } \\
\text { assessing space of } \\
\text { possibilities }\end{array}$ \\
\hline $\begin{array}{l}\text { Virtual } \\
\text { experiments }\end{array}$ & $\begin{array}{l}\text { Virtual experiments } \\
\text { can consider the } \\
\text { impact of different } \\
\text { learning or } \\
\text { adaptation schemes } \\
\text { or scheme } \\
\text { consequences under } \\
\text { different conditions }\end{array}$ & $\begin{array}{l}\text { Virtual experiments } \\
\text { can consider the } \\
\text { impact of adding in } \\
\text { different processes }\end{array}$ & $\begin{array}{l}\text { Virtual experiments } \\
\text { can consider the } \\
\text { impact of different } \\
\text { types and levels of } \\
\text { heterogeneity on } \\
\text { different outcomes }\end{array}$ & $\begin{array}{l}\text { Virtual experiments } \\
\text { are used to explore } \\
\text { space of possibilities }\end{array}$ \\
\hline Interoperability & $\begin{array}{l}\text { Learning and } \\
\text { adaptation may alter } \\
\text { interoperability over } \\
\text { the course of the run } \\
\text { if they change the } \\
\text { semantic } \\
\text { composability }\end{array}$ & $\begin{array}{l}\text { Meta-stable } \\
\text { processes means } \\
\text { models of models } \\
\text { may not produce } \\
\text { same results as } \\
\text { models separately }\end{array}$ & $\begin{array}{l}\text { The type and level of } \\
\text { agent heterogeneity } \\
\text { may impact whether } \\
\text { two models are } \\
\text { interoperable }\end{array}$ & $\begin{array}{l}\text { How models are } \\
\text { interoperable will } \\
\text { impact whether the } \\
\text { space of possibilities } \\
\text { that can be explored } \\
\text { increases or } \\
\text { decreases }\end{array}$ \\
\hline $\begin{array}{l}\text { Response } \\
\text { surface } \\
\text { methodology }\end{array}$ & $\begin{array}{l}\text { Fast variant cannot } \\
\text { be used for a system } \\
\text { that has } \\
\text { discontinuous } \\
\text { changes due to } \\
\text { learning or } \\
\text { adaptation }\end{array}$ & $\begin{array}{l}\text { Fast variant valid } \\
\text { only within a stable } \\
\text { range of processes }\end{array}$ & $\begin{array}{l}\text { Fast variant cannot be } \\
\text { used for other } \\
\text { combinations of } \\
\text { agents }\end{array}$ & $\begin{array}{l}\text { Fast variant cannot be } \\
\text { used for examining } \\
\text { space of possibilities }\end{array}$ \\
\hline $\begin{array}{l}\text { Validation in } \\
\text { parts }\end{array}$ & $\begin{array}{l}\text { Model can never be } \\
\text { fully validated }\end{array}$ & $\begin{array}{l}\text { Model can never be } \\
\text { fully validated }\end{array}$ & $\begin{array}{l}\text { Data for validation } \\
\text { may be costly, large, } \\
\text { or only partially } \\
\text { available }\end{array}$ & $\begin{array}{l}\text { Traditional validation } \\
\text { results in overfitting } \\
\text { and makes model less } \\
\text { useful for exploring } \\
\text { space of possibilities }\end{array}$ \\
\hline
\end{tabular}

A final caveat: all models that informed this analysis were information diffusion, belief dispersion social influence models. There are likely to be additional best practices from other problem domains.

\subsection{Dynamic Networks}

Model realism can be increased by using realistic networks of relations, and depending on the time frame of the model, realistic network evolution models. 
Human society is organized around and through networks. Who you know impacts what you know, your education, health, chances of promotion, and much more. These networks are a function of cultural practices, ethnic group norms, family ties, type of occupation, level of education and so much more. The critical point is not just that there are networks which constrain and enable behavior, but that those networks evolve over time. In addition such networks are not just about people. Networks among organizations impact organizational outcomes. Networks among words, impact recall, sense-making, and definitions. Networks among tasks impact how work gets done. Thus, significant realism is missing in artificial societies if there are not networks. Unfortunately, many modeling toolkits define a network only in terms of which agents are in the same or nearby locations (such as on a grid), or they treat the networks as predefined and static even when the time frame of the model is such that human networks would have evolved.

Models which allow for network based interaction with dynamic networks are of greater utility in reasoning about the spread of disease, the diffusion of information and belief, and the flow of resources. Networks are a "meso" device that supports linking micro behavior to macro-behavior and so support multi-level analytics and validation. For example, given a network, agents who come into contact with each other can learn from each other, leading to widespread cultural evolution. Networks can be layered together, e.g., people can be connected by a Twitter network, a face-to-face interaction network, and so forth. Models that employ networks are a natural way of modeling communication technology and so studying the behavior of people on and through such media. Further as network data can be collected and exists widely, models with a network backdrop have aspects that can be readily validated. Agent-based dynamic-network models can be used at all levels; e.g., the agents can be humans (Carley, Martin and Hirshman 2009), decision making units (Carley, Morgan and Lanham 2016), or countries (Lin, Carley and Cheng 2016).

\subsection{Social Cognition}

\section{Model realism and efficiency can be increased by giving agents a social cognitive system.}

Social cognition refers to the cognitive processes and biases that impact how people make sense of the world from a social or group perspective. It includes those mental shortcuts that we have developed to reason about others and make decisions with little more than group level information. This includes those processes for storing and applying information about people and social situations such as generalization, social inference and reasoning about the generalized other.

In the agent-based modeling area, models tend to either use rich high realistic cognitive models or very simplistic (just a few rules) cognitive models. In general, the more veridical the cognitive model the fewer agents are modeled; whereas, in most artificial society models there are huge numbers of cognitively simplistic agents. However, adding social cognition is a win. The model results will be a better fit to human behavior, and the model will run faster. Social cognition as a cognitive shortcut means that as humans we need to process and store less data; and it means that artificial agents with social cognition need to process and store less data (Morgan, Joseph and Carley 2017). Hence, by adding social cognition you can have more cognitively accurate agents and more agents.

\subsection{Virtual Experiments}

Use virtual experiments to constrain model development, ensure purpose is being met, and to analyze model results.

A virtual experiment is an experiment conducted using a simulation. In this virtual experiment a set of factors are varied and the activities of the artificial society monitored to identify the impact of those factors on the outcomes of interest. As simulation models become more complex, these virtual experiments like experiments in the real world, do not capture all features of the society (artificial or real), and so care must be taken to control for key potentially confounding variables.

Models are typically built with a purpose in mind. This purpose can be expressed as one or more questions that the model will be used to answer. These questions may center around the viability of alternative 
theories, or the potential response of the system to alternative hypothetical future scenarios. The way these questions will be addressed is formalized in the design of the virtual experiment. Laying out the virtual experiment provides guidance for what factors must be modeled, and what factors are out of scope. Laying out the virtual experiment provides a way to estimate the cost of the analysis and the time it will take to generate meaningful model results.

Virtual experiments are the key to how the results from models are assessed (Maxwell and Carley 2009). The execution of a virtual experiment requires serious thought and planning. The quality of the virtual experiment's design and analysis are as critical to the models utility as the entire model development process. In planning a virtual experiment, the analyst must select a set of independent variables that are relevant to the policy alternatives or hypotheses that are under consideration. Levels or values for each of these variable that are representative or critical must be chosen. These values should cover the range of possibilities for the independent variables and so sample the entire space of feasible possibilities. In addition, interim values to allow for the possibility of identifying trends, and points of inflection in response surfaces should be included. Dependent variables should be chosen based on policy or scientific concerns. Standard experimental design constructs such as Box-Behken can be used; however, which is used depends on the virtual experiment. However, the number of variables in artificial society models may be quite large and may include non-continuous variables. In that case many of the experimental design constructs devised for physical device simulations may not be appropriate. Cells in the virtual experiment should be pre-tested for computational running time and the overall time to conduct the experiment should be estimated. At that point, it may be necessary to trim the experiment or gain access to more processors. After running the virtual experiment the results will need to be statistically analyzed. It is important to recognize that with virtual experiments, unlike the real world analog, it is trivial to achieve tight confidence intervals and a high R2 simply by increasing the number of replications that are simulated. Hence, statistical significance levels should not be used to indicate significance, but rather as assessments of the relative strength of association. Sensitivity analyses (Gilbert 2008) are thus critical for understanding the meaning and reliability of the virtual experiment's results. Virtual experiments also lie at the heart of validation (Louie and Carley, 2008).

\subsection{Interoperability}

\section{Design for interoperability.}

A simulation model is a piece of software and as such should be interoperable with other pieces of software. At a minimum the model should be designed so that it can accept data input in standard forms and generate output in such standard forms. In many cases this means, ensuring that the program can read and write comma-separated value files. Two models that interoperate at this level can be syntactically composed into a larger model, or model of models. For more mature systems this means ensuring that the model uses data at the same level granularity as other models or that there are appropriate intermediate algorithms for converting between granularity levels. Similar levels of granularity enable semantic composability. Illustrative dimensions where granularity is important are temporal scale (e.g., Minute or year), spatial scale (e.g., city block of country), population or group scale (e.g., person or organization), and knowledge scale (e.g., word to narrative). Interoperability goes beyond matching levels of granularity to linking models at different scales, and fundamentally different logics (Orr et al, 2019 forthcoming).

Interoperability is critical. Designing for and supporting interoperability means that the simulation model can use existing data sets and its output can be analyzed in standard statistical and social network analytic tools. This facilitates validation. This also facilitates analyzing the results of virtual experiments conducted using the model. Interoperability also supports model docking (Axtell et al. 1996) which enables researchers to see whether or not two or more models have an underlying theoretical consistency.

Models are also increasingly used as components in a model of models or multi-model systems (Carley et al. 2012, National Academies of Sciences, Engineering, and Medicine 2016). One such system is where multiple models of the same phenomena are used in conjunction to generate the behavior of the same variables and the results from all models are "averaged" to get an overall assessment. The classic example 
of this is in weather modeling. A second type of multi-model system is where complex systems are modeling by linking together a set of component models, such that each component can be assessed and validated separately. Interoperability supports both types of multi-modeling and so enables practitioners to move beyond the limits of single models, supports re-use, and improved science through triangulation.

\subsection{Measurable Parameters}

\section{Attempt to minimize the number of parameters and use only parameters that can be measured.}

Models generally have a number of parameters. These parameters may take many different forms. For example a parameter may be a feature of a specific rule; e.g., if two agents occupy the same square they exchange tokens. Or a parameter may be a numerical process control; e.g., every $n$ time periods agents update their perception of others. Or a parameter may be the initialization settings; e.g., run the model with agent decision making based on standard operating procedure, use 100 agents, and put agents in a random network. Some parameters may be dictated by the theory that is being instantiated; however, in most cases parameters are created by the modeler to fill in missing gaps in the theory and to ensure that the simulation will run. Nevertheless, these parameters are critical to the way the simulation runs and the results generated.

There are a number of issues around model parameters. First, work toward parameter-less models. Often parameters are added as a programming shortcut rather than taking the effort to model some underlying process. While this can work it also introduces errors. Second, only use parameters that have real-world measurable analogs. Parameters that cannot be measured cannot be validated, weaken the theoretical results, and are a potential unmeasurable source of uncertainty in the model. Third, where possible set parameters based on contextually relevant empirical data. This provides a level of validation to the model

The final point has to do with model tuning or calibration. For physical systems, parameters in the simulation model are often set by calibrating the model so that the data generated fits the data measured from the physical system. Optimization approaches for estimating parameter values provide higher statistical and computational efficiency, enable more complex - higher dimensional - models to be calibrated, and support the user in parameter setting (Talpaz, DaRoza and Hearn 1987). This type of activity is often viewed as a necessary form of validation. However, this approach, for socio-cultural models often results in overfitting and reduces the ability of the model to forecast future events. For example, BioWar was shown to produce comparable results to an SIR model and to be valid given the Russian Anthrax release; however, unlike the SIR model BioWar was not overfitted to one set of data and so could be used in other situations such as modeling the Anthrax release in the US Senate office (Chen et al, 2006).

\subsection{Response Surface Methodology}

Employ response surface methodology.

Response surface methodology is a statistical approach for exploring the relation between independent and dependent variables. For simulation models, this is done by conducted a sequence of virtual experiments. Then a set of increasingly complex polynomials are fit to the data to characterize the relation. The most parsimonious best fitting polynomial is then treated as the characteristic model of the response surface. This process creates a simplified estimate of how the model operates within certain bounds. As long as those bounds hold, the equation derived by this methodology can be used, in lieu of the model, to estimate the model solution to given variations in those independent variables.

Agent-based models can require significant time and storage to run. For a developed validated model, response surface modeling can be valuable for creating a reduced form of the model subject to particular boundary conditions. This reduced form, which is the parsimonious best fit polynomial, can within scope conditions permit rapid and scalable simulations. Response surface methodology also provides a way of linking simulation to statistical modeling and so provides a way of explaining model results to those not comfortable with simulation. Finally, the reduced forms of multiple models can be compared to identify critical differences in the models. The negative aspects of response surface modeling is that it is typically 
used only to relate inputs to outputs at one time period. If you are interested in model dynamics, and not just the final outcome, then the response surface needs to be re-estimated at each time period or a lagged regression model is needed. This added complexity is often prohibitive.

\subsection{Validation in Parts}

\section{Employ a level of validation appropriate to the model purpose and use validation in parts.}

The science of validation for simulation models was initially developed in the context of shop floor management and operations research. The goal was to model physical systems, and to validate the models against data from real physical devices. A key assumption for the science of validation is stationarity of process and, the corollary, components in the system do not learn or adapt. This assumption is violated in social-behavioral systems. This makes the traditional approach less applicable. A new science of validation for adaptive social systems is needed (National Research Council 2006, National Research Council 2008).

While this new science has yet to emerge, some key features and practices have emerged. Four of these are considered. First, use model-to-model comparison. Demonstrating that multiple models generate similar results, despite being based on fundamentally different theories, is considered a validation of the robustness of the result. Second, tuning can be overfitting. As noted earlier, validation by tuning to historical data runs the risk of overfitting the model, and may reduce the model's ability to forecast changes. Third, fit data to model not model to data. To reduce overfitting demonstrate that multiple historical data points are within the range of possibilities generated by the model, and not that the model results are within the empirical range of the real world data. Fourth, conduct validation in parts. Validation in parts refers to the practice of validating each of the three key model elements separately so as to reduce overfitting (Carley et al. 2012). These elements are input, internal processes, and outputs. For model input, validation is achieved if it matches at least the distributional properties of the real world data; although, it is even better if the input data is actually real world data. For internal processes validation is achieved if model processes lightly dock with the theory or the process being modeled. In general, internal process validation is often a combination of match to theory, match to lab experiments, and match to field observations all of which may be done at different levels of granularity from the qualitative to point level quantitative predictive level. For the model outputs, the classic approach, and that used in physical or mechanical system modeling, is that the simulation outputs are validated by comparison with historical data. For social-behavioral systems, such comparison may actually lead to overfitting. Hence, it is generally better to show a match against multiple historical data points with some level of error, than a perfect match with one historical data point.

Validation in parts is distinct from semantic composability; however, it is predicated on the idea that in some cases it will be possible to validate sub-models separately when building a model of models. Building a valid model of models requires syntactic and semantic composability (Petty and Weisel, 2003). Composability supports model reuse. Syntactic composability means that the sub-models have been coded for interoperation and so can pass data from one model to the next e.g., there is a good API. Semantic composability mean that the sub-models can be meaningfully combined he data that is passed is what the kind of data expected; e.g., both models expect time in the form of months. Composability requires there to be multiple sub-models; validation in parts does not. In a model of models, even when both syntactic and semantic composability are present, each of the sub-models may have been validated using a validation in parts approach where the inputs, processes and results are validated separately.

Another point is that the way historical data and simulated data are matched is reversed for physicalmechanical systems and social-behavioral systems. For physical-mechanical systems the question being addressed is - are the simulated results drawn from the same underlying distribution as those observed in the real world? Typically one has many historical points, and can generate distributions over time of for any point in the history of the system being modeled. Statistical tests are run to see if model behavior could lie in the distribution observed in the real world. A well matched model can then be used for prediction. 
In contrast, for social-behavioral systems the question being address is - does the historical data lie within the distribution described by the simulation? In this case, there is generally a single historical point and a virtual experiment is run using the simulation model to estimate a response surface. Statistical tests are run to see if the real observations lie within the distribution observed from the model's results. A well matched model could then be used to explore the space of future possibilities.

Note, in general this can get very messy for social behavioral models. Data for the historical case is typically incomplete and/or at different levels of granularity. When there are multiple historical cases there are often discontinuities in the underlying conditions that cause the historical cases to not be completely comparable. Nevertheless, as such discontinuities can be modeled, matching is still possible. Logically, one would expect that as the number of historical cases increases, the distribution of historical data points should come to reflect the plausibility distribution from the simulation.

Sometimes social-behavioral models are best validated by seeing if the patterns that they produce are close to those observed in the real world. One approach for doing this is to measure the distance between the group-level networks observed from both the simulated and real data. In this case, socio-cultural cognitive mapping (Morgan, Levine and Carley, 2017) can be used to generate the best-fit network model from the real data, and then again from the simulated data. This elevates the analysis from the agent level to the group level. Then network comparison techniques can be used to assess the extent of network similarity.

Finally, once the model's purpose and the nature of the data available for validation are considered the conclusion may be that it is not possible to fully validate the model empirically. The advantage of validation in parts is that different levels and types of validation can be used for different parts of the model. For example, the input data that is used to instantiate the model may be based on current data derived from empirical case studies. The internal processes might be validated only at the face validity level, others might be validated against lab experiments, and still others left as random process assumptions as they are not critical to the model's purpose. The model results may be validated by showing that a case study of data fits within the distributions generated by the model, and/or by showing that when the model is used to simulate an existing system the results match a set of stylized facts.

To further illustrate the value of validation in parts it is worth contrasting simulation and machine learning models. Machine learning models are generally developed to categorize data, such as -- is a Twitter account a bot or not. This categorization when applied to new data is called prediction. These models are trained using "ground truth data"; i.e., data in which the items being categorized have been previously marked for their category. The strength of these models comes from the vast quantity of data that can be used to train and test the model. Accuracy with respect to predicting items where the ground truth is known is used as a measure of model validity. These validated machine learning models can then be used in other very similar settings where the data is known to have characteristics comparable to the training data.

In contrast, simulation models, particularly ABMs of social-behavioral systems, are generally developed when the user wants to investigate a phenomena where ground truth is either not known, or where there is very little data. Thus there is not data for both training and testing. These models can be validated not through a train/test procedure but through calibration i.e. showing that the single real data point fits within the set of possible outcomes from the simulation. Once validated, the simulation model is then used to explore a series of scenarios that are futuristic, or are purely hypothetical. For example, the social simulation model is used not for determining whether or not a Twitter account is a bot, but whether or not changes in Twitter's policies about following, account verification, or message prioritization will change the reach of a bot's messaging strategy. Validation in parts must be used because none of these alternative scenarios have ever happened so there is no ground truth data against which to do train/test style of validation.

\section{CONCLUSION}

Artificial societies support reasoning about social-behavioral systems. Their strengths are many, including admitting the level heterogeneity observed in the real world, and supporting reasoning about process rather 
than just end-states. A key value of these models though, is that they support theory development (Davis, Eisenhardt, and Bingham 2007). Increasingly these models are coming to be predicated on better models of human cognition (Sun 2006). Further, these models are increasingly tied to real data, and may even sit on top of real-time data streams. Despite these welcome advances, social-behavioral modeling is difficult and is used for a class of problems for which historical methods do not work well (Davis et al. 2017).

The heart of many social-behavioral models is that the agents being simulated adapt or learn. The learning sub-modules in artificial societies have traditionally been based on either accurate and detailed cognitive models or light weight models predicated on some form of learning by being told or stochastic reinforcement learning. To be sure more complex models, such as simulated annealing, have been used (Carley and Svoboda 1996). With the growth in machine learning has come a move to incorporate machine learning algorithms as the model of how an agent learns (Macal and North 2010). This approach has merit, however, it is problematic if the model is meant to reflect human learning. Moreover, it is important to bear in mind that machine learning algorithms are not a substitute for artificial societies if the goal is to understand and reason about how people might respond to new and never seen before events. Machine learning models essentially treat tomorrow like today and cannot learn. Rather, they represent the result of learning a particular pattern. If the goal is to reason about the process, and not the end-result, or if the goal is to understand how people might behave, artificial societies will need other types of learning modules.

As we move to the next generation of artificial society based science, researchers in this area will need to focus on issues of bias and validation. Research is needed to create standard and reusable representations. New technologies supporting interoperability and data-enabled testing are needed. Most critically, as a community we need to work toward a new science of validation.

\section{ACKNOWLEDGMENTS}

This work was supported by the Office of Naval Research (ONR) through MINERVA grants N000141310835 on State Stability and N000141512797 on Dynamic Statistical Network Informatics, and by the Defense Advanced Research Projects Agency (DARPA) SBIR Program Office under Contract No. W31P4Q-13-C-0048. The views and conclusions contained in this document are those of the authors and should not be interpreted as representing the official policies, either expressed or implied, of the ONR, DARPA or the U.S. Government.

\section{REFERENCES}

Axtell, R., R. Axelrod, J. M. Epstein, and M. D. Cohen. 1996. "Aligning Simulation Models: A Case Study and Results". Computational and Mathematical Organization Theory vol. 1(2), pp. 123-142.

Bonabeau, E., 2002. Agent-based modeling: Methods and techniques for simulating human systems. Proc. National Academy of Sciences 99: pp. 7280-7

Box-Steffensmeier, J. M., and Jones, B. S., 2004. Event history modeling: A guide for social scientists. Cambridge University Press.

Burton, R. M., and B. Obel. 1995. "Validation and Docking: An Overview, Summary and Challenge". Computational and Mathematical Organization Theory vol. 1(1), pp. 57-71.

Carley, K. M. and G. P. Morgan, 2016. "Inadvertent leaks: exploration via agent-based dynamic network simulation". Computational and Mathematical Organization Theory vol. 22(3): pp. 288-317.

Carley, K. M. and D. T. Maxwell, 2006, "Understanding Taxpayer Behavior and Assessing Potential IRS interventions using Multiagent Dynamic-Network Simulations," In Recent Research on Tax Administration and Compliance: In proceedings of the 2006 IRS Research Conference. Edited by Dalton and Bliss (Eds.), Washington, D.C., pp. 96-103. Available from: http://www.irs.gov/pub/irs-soi/06carley.pdf 
Carley, K. M., D. Fridsma, E. Casman, A. Yahja, N. Altman, L. Chen, B. Kaminsky and D. Nave, 2006. "BioWar: Scalable Agent-based Model of Bioattacks," IEEE Transactions on Systems, Man and Cybernetics-Part A, 36(2):252-265.

Carley, K. M., G. Morgan, M. Lanham, and J. Pfeffer. 2012. "Multi-Modeling and Sociocultural Complexity". In Advances in Design for Cross-Cultural Activities Part II edited by D. D. Schmorrow, and D. M. Nicholson, pp. 128-137. Boca Raton, FL, CRC Press.

Carley, K. M., G. P. Morgan, and M. J. Lanham. 2017. "Deterring the Development and Use of Nuclear Weapons: A Multi-Level Modeling Approach". The Journal of Defense Modeling and Simulation vol. 14.1, pp. 95-105.

Carley, K. M., M. K. Martin and B. Hirshman. 2009. "The Etiology of Social Change”. Topics in Cognitive Science vol. 1.4, pp. 621-650.

Carley, K.M. and D. M. Svoboda. 1996. "Modeling Organizational Adaptation as a Simulated Annealing Process". Sociological Methods and Research vol. 25(1), pp.138-168.

Chen, L., K. M. Carley, D. Fridsma, B. Kaminsky and A. Yahja, 2006. "Model Alignment of Anthrax Attack Simulations," Decision Support Systems, special issue on Intelligence and Security Informatics, 41(3):654-668.

Davidsson, P., 2002. "Agent based social simulation: A computer science view". Journal of artificial societies and social simulation, vol. 5(1).

Davis, J. P., K. M. Eisenhardt, and C. B. Bingham. 2007. "Developing Theory Through Simulation Methods". Academy of Management Review vol. 32(2), pp.480-499.

Davis, P. K., A. O’Mahony, T. R. Gulden, O. A. Osoba, and K. Sieck. 2017. Priority Challenges for Social and Behavioral Research and Its Modeling. Santa Monica, CA, Rand.

Davis,P.K., A. O'Mahony, J. Pfautz (Eds.), 2019. Social-Behavioral Modeling for Complex Systems. Wiley: Hoboken, NY.

Diallo, S.Y., W. Wildman, F. LeRon Shults, and A. Tolk (Eds.), 2019. Human Simulation: Perspectives, Insights, and Applications. Springer: Cham Switzerland.

Edmonds, B., 2005. "Simulation and complexity-how they can relate". Virtual worlds of precision: computer-based simulations in the sciences and social sciences. Lit-Verlag, Münster pp. 5-32.

Gilbert, N. 2008. Agent-Based Models. Thousand Oaks, CA, SAGE Publications.

Lanham, M. J., G. P. Morgan and K. M. Carley, 2011, "Data-Driven Diffusion Modeling to Examine Deterrence," In proceedings of IEEE NSW 2011, 1st International Workshop on Network Science, West Point, New York, June 22-24, 2011, pp. 1-8.

Lin, L., K. M. Carley, and S. Cheng. 2016. "An Agent-Based Approach to Human Migration Movement". In Proceedings of the 2016 Winter Simulation Conference, edited by T. M. K. Roeder, P. I. Frazier, R. Szechtman, E. Zhou, T. Huschka, and S. E. Chick, eds. Piscataway, New Jersey, Institute of Electrical and Electronics Engineers, Inc.

Louie, M. A. and K. M. Carley, 2008, "Balancing the Criticisms: Validating Multi-Agent Models of Social Systems," in a special issue on "Simulating Organizational Processes" in Simulation Modeling Practice and Theory, 16.2: 242-256.

Macal, C.M. and M. J. North. 2010. "Tutorial on Agent-based Modelling and Simulation". Journal of Simulation vol. 4(3), pp.151-162.

Maxwell, D., and K. M. Carley. 2009. "Principles for Effectively Representing Heterogeneous Populations in Multi-Agent Simulations". In Complex Systems in Knowledge Based Environments edited by A. Tolk, Ch. 8, pp. 199-228. New York, Springer-Verlag.

Mezzour, G., W. Frankenstein, K. M. Carley and L. R. Carley, 2018, A Socio-Computational Approach to Predicting Bioweapon Proliferation, IEEE Transactions on Computational Social Systems 5(2) p. 458467. 
Mohaghegh, Z., and Mosleh, A., 2009. "Incorporating organizational factors into probabilistic risk assessment of complex socio-technical systems: Principles and theoretical foundations". Safety Science, vol. 47(8): pp. 1139-1158.

Morgan, G. P., J. Levine, and K. M. Carley. 2017. "Socio-Cultural Cognitive Mapping”. In Proceedings of the International Conference Social Computing, Behavioral-Cultural Modeling and Prediction and Behavior Representation in Modeling and Simulation edited by D. Lee, Y. Lin, R. Thompson, and N. Osgood pp. 71-76, Washington DC, Springer.

Morgan, G. P., K. Joseph, and K. M. Carley. 2017. "The Power of Social Cognition”. Journal of Social Structure vol. 18, pp. 1-22.

National Academies of Sciences, Engineering, and Medicine. 2016. From Maps to Models: Augmenting the Nation's Geospatial Intelligence Capabilities. Washington, DC, The National Academies Press.

National Research Council. 2006. Defense Modeling, Simulation and Analysis: Meeting the Challenge. Washington, DC, The National Academy Press.

National Research Council. 2008. Behavioral Modeling and Simulation: From Individuals to Societies. Washington, DC, The National Academies Press.

Orr, M., C. Lebiere, A. Stocco, P. Pirolli, B. Press and W. G. Kennedy, 2019 forthcoming, "Multi-Scale Resolution of Neural, Cognitive and Social Systems". Computational and Mathematical Organization Theory.

Petty, M.D. and Weisel, E.W., 2003, April. A formal basis for a theory of semantic composability. In Proceedings of the Spring 2003 Simulation Interoperability Workshop pp. 416-423.

Sterman, John D., 2001. "System dynamics modeling: Tools for learning in a complex world". California management review vol. 43 (4): pp. 8-25.

Sun, R. ed.. 2006. Cognition and Multi-agent Interaction: From Cognitive Modeling to Social Simulation. Cambridge, UK, Cambridge University Press.

Talpaz, H., G. D. Da Roza, and A. B. Hearn. 1987. "Parameter Estimation and Calibration of Simulation Models as a Non-linear Optimization Problem". Agricultural Systems vol. 23(2), pp.107-116.

Van Dam, K. H., Nikolic, I., an Lukszo, Z., (Eds.), 2012. Agent-based modelling of socio-technical systems (Vol. 9). Springer Science \& Business Media.

\section{AUTHOR BIOGRAPHIES}

KATHLEEN M. CARLEY is a Professor of Societal Computing at Carnegie Mellon University and the CEO of Netanomics. She holds a PhD in Sociology from Harvard. Her research interests lie in apply network science and simulation to address real world complex socio-technical issues such as counterterrorism and the spread of fake news. Her email address is kathleen.carley@cs.cmu.edu. 